\title{
¿Cómo deben influir los valores en la ciencia?*
}

\author{
- Hugh Lacey \\ Swarthoore College/Universiade de São Paulo
}

Traducción de LUIS Alvarenga

Los científicos hacen juicios de valor todo el tiempo. Sopesan sus teorías. Se preguntan: ¿Esta teoría se ve confirmada gracias a la evidencia? ¿Posibilita nuevas previsiones y explicaciones de fenómenos importantes? ¿Es consistente con otras teorías ya aceptadas? Las anteriores son preguntas acerca de la aceptabilidad de una teoría, sobre su valor cognitivo (epistemológico, racional). Los criterios de evaluación que las preguntas sugieren -adecuación empírica, poder de predicción y de explicación, etc. - se designan como «valores cognitivos» (Lacey, 1998: cap. 3). Existe cierta controversia en torno de cuáles deberían ser los valores cognitivos apropiados —empero, no está en debate el que los valores cognitivos tengan que cumplir un papel central en la práctica científica-.

Una teoría puede ser evaluada también de otros modos. ¿Es útil? ¿Para qué fines? ¿Puede aplicársele para resolver un problema práctico? ¿Sus aplicaciones pueden servir a los intereses de determinados valores morales y sociales? ¿Sirve a los intereses personales (del investigador)? ¿Resulta consistente en tanto un punto de vista ideológico o religioso? Estas son preguntas acerca del valor social, político, económico o personal de una teoría.

* El presente ensayo forma parte de un libro que publicará la Universidad Federal de Rio Grande do Sul, en Porto Alegre, Brasil. Su contenido fue preparado con el apoyo (parcial) del National Science Foundation de los Estados Unidos (SES-9905945), de la FAPESP y del Swarthmore College. La versión portuguesa se escribió con la asistencia inapreciable de Maria Ines Lacey; y el apartado sobre Galileo, con la colaboración de Pablo Mariconda (Departamento de Filosofia. FFCLH de la USP).

¿Cómo deben influir los valores en la ciencio? 
Dentro de la tradición de la ciencia moderna se ha afirmado, reiteradamente, que los valores cognitivos son distintos de todos los demás tipos de valores, y que sus papeles deben mantenerse separados (Lacey, 1999b). Específicamente, aquellos valores que no son cognitivos no deben influir fundamentalmente sobre la metodología científica. Con todo, los valores sociales y morales, así como otro tipo de valores que no son cognitivos, podrían desempeñar varios papeles en la ciencia (Lacey, 1999a: cap. 1) - por ejemplo: con respecto a la ética de la investigación experimental (Lacey, 1999c), las aplicaciones prácticas, así como todo aquello que se refiera a la elección de los problemas específicos que serán investigados; pero estos valores no deben influir en las valoraciones cognitivas de las teorías, o en las características fundamentales de la investigación básica.

Ochenta años atrás, Poincaré resumió esta perspectiva con las siguientes palabras:

Los dominios propios de la ética y de la ciencia pueden entrar en contacto uno con el otro, pero nunca se penetran mutuamente. La primera nos da la meta, mientras que la segunda nos enseña cómo alcanzarla. Nunca entran en conflicto porque nunca se encuentran. Una ciencia inmoral sería tan imposible como una moral científica. (Poincaré, 1920/1958: p. 12)

Discutiré en esta ocasión, en primer lugar, la propuesta de que los valores (no cognitivos) y la ciencia no se deben interpenetrar - el que esos valores no deben influir en la valoración cognitiva de teorías, o en la dirección fundamental de la investigación básica. En segundo lugar, discutiré una propuesta que se une a la primera: que la ciencia debe conducirse libre de la interferencia de aquellos que no aceptan la primera propuesta y quieren subordinar los empresas de la ciencia a intereses que representan valores sociales, morales, políticos o religiosos.

Esas dos propuestas, ampliamente defendidas y frecuentemente reinterpretadas, se nutren con ciertos casos que se han vuelto símbolos muy conocidos - como los de Galileo y de Lysenko. Simbolizan caminos erróneos que pueden tomarse cuando no se respeta la libertad de la investigación científica. Del caso de Galileo: los errores se perpetúan, la investigación se estanca, se truncan vidas y carreras creativas, se violan derechos humanos; del caso de Lysenko, podríamos destacar lo siguiente: se descartan las aplicaciones deseables, mientras que aquellas alternativas de inspiración ideológica ganan hegemonía, con consecuencias catastróficas. Al reflexionar sobre estos símbolos, se vuelve aparente, porque la propuesta de que la ciencia y los valores (no cognitivos) no se deben de interpenetrar, tenía que vencer en una lucha contra intereses poderosos. No sería sorprendente, pues, si las interpretaciones de la propuesta mostraran señales de lucha. La reivindicación de la libertad de la investigación tiene dos fuentes: (1) El deseo de evitar nuevos casos como los de Galileo y Lysenko; y (2) un ideal de entendimiento científico y de su base racional. Ambas fuentes producen tensiones. Voy a argumentar que actualmente la propuesta de no interpenetración encubre el hecho de que ciertos 
valores sociales, relacionados al control de objetos naturales (Lacey, 1998a: cap. 5), afectan medularmente a las principales empresas de la ciencia.

Cuando discutimos la libertad de investigación científica, Galileo no es tan sólo un símbolo. Sus argumentos a favor de la autonomía de la ciencia son fundamentales. En la primera parte de esta conferencia (que se vale ampliamente de materiales seleccionados de Lacey y Mariconda, 2000) presentaré detalladamente las concepciones de Galileo. Después, en la segunda parte, expondré mis críticas que constituirán el punto de partida para una respuesta positiva a la pregunta: ¿Cómo deben influir los valores (no cognitivos) en la ciencia?

Voy a interpretar el concepto de autonomía de forma sumamente amplia. Entendamos por ella el hecho de que las prácticas científicas se deben conducir libres de toda interferencia externa; y, al mismo tiempo, que dichas prácticas se deben patrocinar con los recursos necesarios provenientes de varias instituciones públicas y particulares — de tal manera que los científicos puedan seguir su objetivo de lograr y de confirmar la comprensión de fenómenos del mundo- de cualquier manera que consideren apropiada (Para una discusión más detallada de la autonomía, ver Lacey, 1999a: caps. 4 y 10).

Típicamente, se recomienda evitar las siguientes interferencias externas: Opiniones religiosas, políticas, ideológicas, populares o interesadas; puntos de vista valorativos y sus presupuestos, y ciertas perspectivas metafísicas. Es claro que los ítems de esta lista evocan antiguas luchas. Talvez se excluyan otros ítems, simplemente porque han sido aliados de la ciencia en el curso de todas sus batallas. (La metafísica materialista (Lacey, 1998: cap. 1) o las visiones matematizadas del mundo ven a la mente y los intereses dados por las aplicaciones del conocimiento científico como posibles aliados).

Galileo no consideró la autonomía en su generalidad. Su meta era conseguir que la investigación científica estuviese libre de la interferencia específica de la iglesia católica ejercida a través de su Magisterium, de su sistema legal con sus condenas y castigos y de otras innumerables imposiciones irritantes; positivamente, su meta era conquistar para los científicos el derecho de investigar, de hacer nuevos descubrimientos y sus propias interpretaciones y valoraciones de los resultados (Mariconda, 1997). Galileo resintió profundamente la interferencia constante en su empresa científica —no sólo porque sus oponentes no admirasen sus notables capacidades y su extraordinaria contribución científica, sino también porque él los despreciaba. Al hablar de sus críticos, dijo lo siguiente:

...creen que se pueden encajonar a todos los filósofos de envergadura entre cuatro paredes. Yo creo que ellos tienen alas y que vuelan como el águila solitaria, y no en bandadas, como auras. Un águila, siendo rara, se deja ver 
poco y se deja oír menos, mientras que las auras se abren campo con su graznar bullicioso y ensucian el suelo cuando se posan sobre él. (Galilei, 1623/1957: p. 239),

Este tipo de retórica era motivo de celebración para sus aliados, aunque también le había granjeado enemigos. Pero la retórica no debe opacar el hecho de que, para Galileo, la autonomía de la ciencia no se fundamentaba en la autocomplacencia, sino en argumentos sustentados. Galileo quiso desarrollar un argumento que fuera tan efectivo como correcto: quiso persuadir a las autoridades religiosas. Cualquier argumento efectivo de que la ciencia debía estar libre de toda interferencia eclesial, no podía permitir que la empresa científica estuviese sujeto a restricciones por parte de sectores opuestos a la iglesia. Galileo tenía que argumentar que la ciencia debe quedar libre de todas las interferencias de afuera; tenía que retratar a la ciencia como un "valor universal", un objeto de valor para cualquier punto de vista moral o metafísico razonable.

El argumento de Galileo - refinado, generalizado y complementado- ocupa un lugar central en las defensas de la autonomía de la ciencia y de la propuesta de que la ciencia y los valores (no cognitivos) no se deben de interpenetrar. La autonomía es necesaria, porque la ciencia y los valores (no cognitivos) no se interpenetran. Podemos reconstruir el argumento de Galileo (desarrollado en varios trabajos, especialmente en Galilei, 1613/1989; 1615/1957; 1632/1962) basándonos en los siguientes tres supuestos:

1. Las iniciativas científicas posibilitan descubrimientos sobre los fenómenos naturales, descubrimientos que son hechos por medio de los propios métodos de la ciencia. Los métodos enfatizan principalmente observaciones e inferencias involucrando a las observaciones. Las cuestiones de valores no lienen nada que ver con la observación precisa o la inferencia válida. Entonces, los métodos de la ciencia no deben responder cognitivamente a las propuestas ni a las críticas hechas desde cualquier punto de vista que involucre valores - ni los de la iglesia, ni los de perspectivas religiosas y morales contrarias a las declaraciones de la iglesia. $\mathrm{Ni}$ la iglesia, ni sus oponentes, ni cualquier punto de vista de valores tienen autoridad sobre el dominio propio de la ciencia.

2. Los juicios científicos son hechos por "especialistas" - aquellas "águilas" que vuelan solitarias-, es decir, científicos con talento intelectual relevante, formados en estos métodos y que han cultivado las virtudes apropiadas para dedicarse a usarlos estrictamente.

3. Los juicios científicos bien establecidos no pueden contradecir - ni aportar evidencias a favor de-cualquier asunto dentro de la esfera de autoridad de la iglesia. Podemos generalizar esta suposición: racionalmente, estos juicios no tienen ninguna implicación en los dominios de la teología, de la metafísica y de los valores; ni aportan evidencias ni argumentos a favor (o en contra) de cual- 
quier punto de vista fundamental dentro de esos dominios, sean de la iglesia o de sus oponentes.

Cada una de estas tres suposiciones, que ahora entraré a discutir en detalle, puede verse como destinada a refutar una objeción hecha por los críticos de Galileo.

1. Imparcialidad. Los juicios científicos, adecuadamente fundamentados, se derivan de observaciones (auxiliadas frecuentemente por el uso de instrumentos que "expanden la percepción", o hechos en los espacios experimentales) e inferencias en que estas observaciones desempeñan un papel importante.

El propio Galileo no entendía bien la inferencia científica; nunca renunció definitivamente a la tradición de la analítica posterior, el opus magnum de la filosofía aristotélica, que sostenía que para aceptar las teorías deben demostrarse con necesidad y certeza (ver las referencias en Lacey y Mariconda, 2000). A pesar de eso, cuando Galileo argumentaba a favor de Copérnico, mostraba una consciencia sutil de los criterios no demostrativos, los valores cognitivos (o, cuando menos, alguno de ellos), que deben dar forma a la inferencia científica bien lograda. Para ilustrar este punto, citaré cuatro de los criterios que Galileo emplcaba explícilamente en sus argumentos.

(i) Adecuación empírica, es decir, concordancia con los fenómenos y los expcrimentos observados, especialmente con los datos empíricos cuantitativamente exactos, y el poder de predicción tocante a estos datos. Nótese que Galileo hacía énfasis en que el método científico requiere investigaciones que aumenten el alcance de los datos relevantes y disponibles. Para él, los datos empíricos sustituyen el "testimonio de autores" (Galilei, 1623/1957: p. 259) y la "autoridad de las escrituras" (Galilei, 1615/1957, p. 182), que eran importantes para sus críticos, y vuelven cosa irrelevante las opiniones del hombre común.

(ii) Poder explicativo, es decir, la capacidad de explicar los fenómenos y clatos observados, la capacidad de identificar las causas de los fenómenos. Aunque en la tradición aristotélica, el análisis causal cstaba ligado íntimamente con la demostración, en la práctica, Galileo se conformaba con explicaciones que luesen superiores a las que sus rivales formulaban. También reconocía que las explicaciones causales involucran relaciones lógicas (entre los datos y las suposiciones teóricas fundamentales), las cuales son mediadas por varias "hipótesis auxiliares". Proponía que tales mediaciones se deben sujetar al siguiente criterio:

(iii) Limitación del uso de las "ficciones", esto es, de las hipótesis ad hoc que Galileo llamaba "disculpas infelices e indigentes" y "subtcrfugios" (Galilei, 1632/1962: p. 317-8) — es decir, minimización del uso de hipólesis introducidas únicamente para mantener la adecuación empírica, en detrimento del poder explicativo. En vez de esas "ficciones", propugna el uso de hipótesis confirmadas (aunque fucra en parte) en virtud de la existencia de analogías experimentales (p. 421).

¿Cómo deben influir los valores en la ciencio? 
(iv) Simplicidad: Armonía - las partes se encajan unas con las otras armoniosamente, con un mínimo de suposiciones. La simplicidad era muy importante para Galileo (y para Copérnico), pero por falta de tiempo no discutiré sobre ella en esta oportunidad (Galileo, 1632/1962: p. 341; Finocchiaro, 1997: p. 328 ff; Mariconda, 1997).

Estos criterios - ejemplos de valores cognitivos- son distintos de otros tipos de valores, inclusive, los valores sociales y religiosos (Lacey, 1999 a: cap. 9, 1999b). Galileo mantenía que una teoría que satisfacía bien esos criterios es superior a otra que no los satisfacía. Pensaba que la teoría copernicana era superior en este sentido a las rivales de aquel tiempo y por lo tanto debía ser aceptada. De hecho, se equivocaba. La teoría de Kepler era superior. No obstante, Galileo hacía el tipo correcto de inferencia científica (aunque él mismo no distinguía claramente sus características entre esas demostraciones) - inferencia que produce conclusiones abiertas a la revaloración, a la luz de observaciones adicionales y de nuevos argumentos basados en valores cognitivos. De esta manera, Galileo anticipó la tesis que, en mi libro Is Science Value Free? (Lacey, 1999a) designo con el nombre de imparcialidad: Acéptese una teoría si, y solamente si, ella manifiesta los valores cognitivos en alto grado, siempre en un grado superior a los de las teorías rivales.

\section{El ethos científico}

Para Galileo, los juicios hechos con arreglo a la imparcialidad derivan en un conocimiento superior a la luz de los criterios, sin tener marca alguna de compromisos religiosos o valorativos. Esto es esencial para su argumento a favor de la autonomía -ésta es necesaria para lograr y confirmar más conocimientos satisfaciendo la prueba de la imparcialidad. El argumento, empero, pretendía legitimar la provisión de libertad a los investigadores como el propio Galileo y Copérnico. Ellos merecen semejante libertad, no sólo en función de su objetivo de hacer juicios imparciales expresando el conocimiento de fenómenos naturales, pero también porque con ellos se puede dar por descontado que los harán habitualmente: son "especialistas" (Galilei, 1632/1962: p. 290) bien formados, que han cultivado las virtudes de la apertura con visión amplia ("open mindedness") y de la racionalidad (Finocchiaro, 1997: p. 340-1). La primera virtud se refiere a la disposición para alcanzar sus conclusiones en forma desinteresada,

- sin dar preferencia a sus propias innovaciones (Galilei, 1615/1957: p. 212), a la luz de mejores argumentos después de haber considerado toda evidencia relevante; aquellos que son abiertos (con visiones de largo alcance) tienen el cuidado de conocer las perspectivas y los argumentos de sus oponentes y de responder, resolutamente, a sus argumentos más fuertes. La segunda virtud se refiere a la actitud (y a la práctica) de aceptar una teoría sólo después de la evaluación completa de los argumentos, en pro y en contra, para verificar si concuerdan con la imparcialidad. 
Galileo sugería que el cultivo de estas virtudes confiere una ventaja metodológica (Finocchiaro: 1997: p. 339). En la controversia sobre la teoría de Copérnico, es claro que los seguidores del astrónomo polaco, tales como Galileo, habían ganado aquella ventaja -inicialmente, la mayoría de ellos eran seguidores de Aristóteles y de Ptolomeo; se convirtieron cuando fueron "inducidos y persuadidos por la fuerza del argumento" (Galilei, 1632/1962: p. 128) - de tal manera que se familiarizaron enormemente con los dos lados de la controversia (incluso, con los mejores argumentos contra Copérnico). En contraste, nadie - después de haber venido a conocer los detalles del argumento favorable a Copérnico- se convirtió al otro lado. Entre los oponentes de Copérnico, lejos de hallar estas virtudes, encontramos ignorancia (resistencia a aprender los argumentos copernicanos), la tendencia a recurrir dogmáticamente a autores antiguos, oportunismo y servilismo. En la perspectiva de Galileo, las virtudes del científico rechazan tales características (vicios) de sus oponentes. (Recuérdese aquí la metáfora del águila y de las auras).

Un águila, y no una bandada de auras, merece autonomía. El argumento a favor de la autonomía supone que los científicos se han vuelto partícipes del "ethos de la ciencia" (Cupani, 1998). La certificación formal de competencia no es algo suficiente para legitimar la autonomía, porque los científicos calificados pueden subordinar sus juicios a los intereses externos. Nadie sabe lo que Galileo hubiera pensado de los hechos contemporáneos: de que ciertos científicos calificados participan en investigaciones controladas por autoridades militares ("classified research"); que ciertas investigaciones se subordinan a intereses empresariales; que la certificación formal podría ligar la investigación científica firmemente a un punto de vista metafísico, excluyendo del dominio de la ciencia investigaciones científicas que podrían tener un gran significado práctico; y que, generalmente, las instituciones de la ciencia se conforman frente a estos hechos. Haré de lado toda especulación a ese respecto. Con todo, el argumento de Galileo a favor de la autonomía depende de los científicos que quieren hacer juicios imparciales y que cultivan las virtudes generalmente necesarias para asegurar el éxito de estos.

\section{El argumento de los “dos libros".}

Siguiendo a Galileo, sería imposible que ocurra un conflicto congitivo (epistemológico) real entre resultados científicos y verdades religiosas (inclusive, de las escrituras). La verdad representa una armonía mutua, pero no una subordinación de un dominio de verdad por encima de otro. Galileo introdujo una nueva manera de explicar por qué eso es necesario (Lacey y Mariconda, 2000): lo importante, al respecto de cualquier enunciado, es preguntar —usando su sugestiva metáfora-: ¿En qué libro esta escrito? ¿En el libro de la naturaleza o en el libro de la revelación (Blackwell, 1991: ch. 7; Mariconda, 1997)? Estos libros, autorizados por Dios, están escritos en lenguajes diferentes, usando (en un.grado significativo) categorías inconmensurables; los libros sirven a fines

¿Cómo deben influir los valores en la ciencia? 
diferentes y necesitan ser leídos en formas diferentes - no pueden ser traducidos uno al otro: por lo tanto, nunca podrían contradecirse.

La filosofía está escrita en ese libro grandioso, el universo, que se mantiene abierto a nuestra vista. Pero el libro no puede entenderse a no ser que primero se aprenda el lenguaje y se lean las letras con que fue compuesto. Fue escrito en el lenguaje de la matemática y sus caracteres son triángulos, círculos y otras figuras geométricas sin las cuales es humanamente imposible entender una sola palabra; sin entender esos caracteres, intentarlo es como deambular en un laberinto oscuro (Galilei, 1623/1957: p. 237-8)

El lenguaje de la ciencia —el libro de la naturaleza- son las matemáticas, rigurosas y exactas. La Biblia —el libro de la Revelación- está escrita en lenguaje común, abierta al entendimiento común de los legos, describiendo apariencias, algunas veces ambiguas o imprecisas, frecuentemente metafóricas. Hay dos lenguajes: no hay dos mundos distintos ni dos dominios distintos de fenómenos: dos lenguajes que frecuentemente se aplican a los mismos fenómenos — pero, cuando ambos se aplican, reflejan intereses distintos. Cuando una persona cualquiera dice que el sol se mueve a través del cielo, describe lo que observó sin intentar explicarlo; no se pregunta si, realmente, es el sol o la tierra la que está en movimiento. Independientemente de la respuesta "correcta", lo que se ha visto permanece igual. Ambos lenguajes pueden emplearse para describir "los hechos"; la aceptación científica de la teoría copernicana no contradice la relación derivada de la observación común de que el sol se mueve del este al oeste. La Biblia emplea un lenguaje común, suficiente para relacionar fenómenos a la historia sagrada de la salvación; y de esta manera no se dispersa abordando cuestiones científicas (por ejemplo, sobre explicaciones de fenómenos comúnmente observados). Por otro lado, para Galileo, la actividad científica y sus descubrimientos no contribuyen a iluminar el sentido de la historia de la salvación. (Con todo, la actividad científica tiene importancia en el dominio de la salvación, al ser una respuesta a una vocación de leer el libro de la naturaleza, y así llevar a conocer mejor la creación divina).

El argumento de los "dos libros" presupone que los juicios científicos bien hechos concuerdan con la imparcialidad, esto es, que los criterios interpretativos de la naturaleza son distintos de aquellos que le pertenecen a la Biblia, y que los juicios científicos se hacen actualmente con arreglo a los métodos propios de la ciencia, sin ninguna restricción de factores "de fuera". El carácter técnico del lenguaje científico es importante aquí —-matemáticas: exactas, abstractas; por lo tanto, su lenguaje es adecuado para representar la ley, la estructura, la interacción y el proceso subyacentes en los fenómenos. De esta manera, el lenguaje de la ciencia no contiene ni las categorías de valor ni otras categorías implicadas en la experiencia humana y en las relaciones sociales. Así, el lenguaje que la ciencia exige no puede emplearse normalmente en la comunicación del discurso común y carece de los aspectos necesarios para el discurso teológico.

\section{6}

Realidad 81, 2001 
El argumento de los "dos libros" se generaliza con facilidad: desde afirmar que el lenguaje de la ciencia carece de las características exigidas por cualquier discurso sobre valores, hasta aseverar que las teorías científicas no pueden tener ninguna implicación lógica en el dominio de los valores, verbigracia: la ciencia es (en mi terminología) cognitivamente neutra en el dominio de los valores. Así, el argumento de los "dos libros" se generaliza en el argumento de los "discursos múltiples": el discurso de la ciencia y los varios discursos de valores; donde el discurso de la ciencia es inconmensurable con todos aquellos referentes de valores; y así, las teorías científicas no pueden servir de base para favorecer a uno en detrimento de otro. La ciencia no puede resolver las grandes controversias con respecto a los valores.

Se sigue, por lo tanto, que la autonomía presupone imparcialidad y neutralidad cognitiva - que la ciencia y los valores no se interpenetran- y que, de hecho, la actividad científica es conducida por los científicos que han cultivado el ethos científico. (Al mismo tiempo, el argumento de Galileo a favor de la autonomía implica en el reconocimiento de una cierta autonomía de otros discursos, los discursos de valor y el discurso teológico).

\section{II}

Pese a que los argumentos de los "dos libros" y de los "discursos múltiples" han convencido a muchos filósofos y teólogos, enfrentan dificultades. Partir de los "dos libros" presupone:

-Que los juicios científicos se hacen normalmente con arreglo a la imparcialidad; y

-Que el lenguaje de la teoría es "matemático" y "técnico", capaz de representar la ley, la estructura, la interacción y el proceso que subyacen en los fenómenos.

Es el lenguaje técnico, no su concordancia con la imparcialidad, el que asegura que las teorías científicas son cognitivamente neutras.

Pero la neutralidad cognitiva no implica el otro tipo de neutralidad que designaré neutralidad aplicada: en principio, los intereses de todos los puntos de vista de valor razonables podrían servirse de modo igual ("evenhandedly") por aplicaciones científicas. En mi libro argumenté que, en la aplicación, las teorías formuladas en el lenguaje técnico galileano favorecen especialmente a aquellas perspectivas de valor que contienen actitudes específicamente modernas con respecto al control de objetos naturales —o sea, pueden ser aplicadas con mayor prontitud para servir a los intereses ligados al control de los objetos naturales, entendido como un valor no subordinado sistemáticamente a otros valores sociales (Lacey, 1999a: caps. 6 y 10; ver también 1998, cap. 5; 1999d; 2001a).

¿Cómo deben influir los valores en la clencio? 
Estas actitudes hacia cl control, sin cmbargo, están en tensión con (entre olras cosis) la perspectiva de valor que favorece la iglesia católica. Pero, sin la ne'uralidad aplicada, el argumento a favor de la autonomía pierde la deseada fuer á universial. Gillileo no discutió estos asuntos ligados a la aplicación del conocimicnlo cientílico. Sin embargo, en la aclualidad no podemos separar el destino cientilico de sus aplicaciones. Entonces, en la reflexión sobre la autonomía necesitamos desalentar no solamente la ausencia de interferencias externas, sino lambién la disponibilidad de recursos materiales y sociales necesarios para las investigaciones. La falta de neutralidad aplicada no robustece las razones para interferir con los contenidos de los resultados científicos, sino que aquellos cuyos intereses no estuvieran bien servidos podrían cuestionar el valor de patrocinar la investigación "autónoma" que actualmente produce resultados sin manifestar la neutralidad aplicada. (Quienes hacen cuestionamientos de esta índole podrían patrocinar investigaciones conducidas bajo control democrático).

Se hace, así, una pregunta: ¿Por qué aceptar que el lenguaje de la teoría debe ser "matemático" o "técnico?" Una posible respuesta sería: ¡Porque funciona, porque produce resultados! Pero este hecho sustenta tan sólo la autonomía limitada a aquellos dominios de la investigación dentro de los cuales podríamos esperar que la neutralidad aplicada se manifieste. Además de eso, no implica que podríamos obtener resultados únicamente de esta manera. Otra respuesta, afirmada frecuentemente por toda la tradición de la ciencia moderna, es que este lenguaje refleja el mundo tal como realmente es - la ley, la estructura, la interacción y el proceso del mundo son matemáticos, completamente, en todos sus detalles (Lacey, 1998: cap. 1)-. Esta respuesta es metafísica. Galileo hizo alusión a ella; Descartes la desarrolló minuciosamente, y ambos emplearon el artificio del dualismo (mente/cuerpo) para excluir del ámbito del análisis matemático a los fenómenos humanos importantes. La materia (naturaleza) es matemática; la mente (naturaleza humana) no lo es. Obsérvese que este respuesta metafísica depende de la suposición de imparcialidad, y también que la imparcialidad no presupone ni implica la aceptación de cualquier perspectiva metafísica.

Los juicios imparciales pueden hacerse de muchas formas, no solamente en el discurso "matemático" (Lacey, 1999a: caps. 8-10; 1999d). Además de eso, no fue por un resultado científico, aceptado de acuerdo con la imparcialidad, que la naturaleza (abstraída de la mente) es de carácter profundamente matemático. Esta afirmación es una restricción impuesta sobre las teorías permitidas en la investigación científica -y es una restricción de origen metafísico. [Si fuera aceptada, podría explicar la ausencia de la neutralidad aplicada en función de ciertas perspectivas valorativas y religiosas que carecen de acuerdo con la realidad. Y, es cierto, existen contradicciones entre esta metafísica y las presuposiciones de la teología católica - se nota: la contradicción entre la metafísica y la teología, pero no entre la teología y los resultados científicos aceptados de acuerdo a la imparcialidad.] 
Visto de este modo, los "dos libros" equivalen a una defensa de la autonomía de la religión, pero no de las restricciones metafísicas. Sin un argumento convincente por parte de la metafísica, este no sería un argumento serio a favor de la libertad de la ciencia con relación a todas las interferencias de fuera - aún cuando la restricción metafísica sea autoimpuesta por los propios científicos y no impuesta por los poderes de fuera-.

Ahora voy a salirme del contexto proporcionado por Galileo. Pregunto: ¿ Necesitaría emprenderse la investigación científica bajo restricciones de origen metafísico? Si así fuera el caso, ¿por qué? ¿Por qué debería patrocinar un determinado tipo de investigación y participar de ella si yo tuviera una perspectiva valorativa que no se verá favorecida por la aplicación de los resultados de ese tipo de investigación?

Ya indiqué que podemos aspirar a hacer juicios en concordancia con la imparcialidad, sin necesidad de emplear siempre (o únicamente) el lenguaje "técnico" o "matemático", el lenguaje que permite representar fenómenos desde el punto de vista de ley, estructura, interacción y proceso subyacentes. Cuando investigamos, por ejemplo, el comportamiento humano o las patologías psicológicas, ¿por qué emplear el lenguaje "lécnico"? De hecho, actualmente no disponemos de buenas razones para hacerlo (Lacey, 200lb). Talvez pudiéramos obtener mejores explicaciones y anticipaciones de las posibilidades humanas, si empleásemos las categorías intencionales (mentales) del lenguaje común (por ejemplo, creencia o deseo); las categorías de valor, virtud, emoción y sus alcances creadores (tal como en el psicoanálisis); categorías que expresan las variopintas relaciones sociales o el lugar de una persona en una comunidad o en una cultura - o incluso categorías religiosas, como pecado o gracia-.

En verdad, existen "discursos múltiplcs". También es cierto que el lenguaje "matemático" se puede extender de modo indeterminado. Salvo lo que respecta a las ciencias físicas, con todo, no disponemos de argumentos satisfactorios, en el presente, para afirmar que el lenguaje "matemático" describe mejor la realidad de lo que pueden hacerlo otras descripciones en otros lenguajes. En otras palabras, no hay a la disposición ningún argumento bien establccido para aceptar que los estados intencionales y las relaciones sociales puedan reducirse a o sustituirse por fenómenos neurofísiológicos u otros completamente explicables por las categorías conductistas, sociobiológica o cualquier otra categoría "matemática/técnica". Por lo tanto, no hay ninguna razón cognitiva (epistemológica) disponible para no practicar la investigación sistemática y empírica de tal manera que permita el uso de categorías intencionales en explicaciones y tentativas de identificar las posibilidades humanas (Lacey, 2001 b).

Esto es cl comicnzo de un argumento para permitir los "discursos múltiples" dentro de ciertas árcas de investigación científica o de la investigación sistemática y empírica.

¿Cómo deben influir los valores en la ciencio? 
El argumento es convincente, pero con respecto a las ciencias humanas; y ya no empleamos el artificio del "dualismo" (mente/cuerpo) para excluir los fenómenos característicamente humanos del dominio de la investigación empírica. De hecho, en las ciencias humanas se acostumbra encontrar una variedad de abordajes en la investigación —exhibiendo típicamente grados de desarrollo diversos y desiguales, así como relaciones mutuamente conflictivas. El conflicto se deriva, generalmente, del hecho de que cada abordaje tiende a obtener su razón de ser ("rationale") no sólo de ciertas concepciones de los ideales racionales, sino también de sus vínculos con puntos de vista valorativos particulares. Por ejemplo, en la psicología, los abordajes conductistas se ven motivados, parcialmente, por el valor de aumentar nuestra capacidad de ejercer control sobre el comportamiento; mientras que algunos abordajes de psicología cognitiva son motivados, en parte, por un énfasis en el valor de la racionalidad (Lacey, 2001 b; cfr. 1998: cap. 8).

Nótese, empero, que adoptar una perspectiva de valores apenas puede conducir a un abordaje particular; no determina los resultados de la investigación dentro del abordaje, ni robustece las razones para seguir investigando en un abordaje que no produzca teorías que vengan a aceptarse en concordancia con la imparcialidad (Lacey, 1998; 1999a; 1998d ). El abordaje conductista restringe las hipótesis teóricas a aquellas que representan relaciones entre comportamientos y contingencias ambientales y genéticas, y las leyes que gobiernan esas relaciones, pero la investigación empírica necesita establecer cuáles son las relaciones y las leyes actuales, si las hubiese. Bajo la restricción conductista, hay teorías que son aceptadas por satisfacer la condición de imparcialidad. De igual manera, un abordaje de la psicología cognitiva restringe las teorías a la formulación de hipótesis que representan estructuras mentales y procesos computables - pero solamente a partir de la investigación empírica podemos tener esperanzas de identificar correctamente cualquier estructura y proceso. Sin embargo, una vez se respeta la imparcialidad. Ambos abordajes en psicología —el conductista y el cognitivo- producían resultados (teóricos y empíricos) bien establecidos, resultados que son mutuamente consistentes. El conflicto entre los abordajes no deviene en contradicción entre los resultados conductistas y los cognitivos bien establecidos. Lo que está establecido en concordancia con la imparcialidad es el hecho de que ciertos ficnómenos, dentro de espacios específicos, son explicables en función de los principios teóricos de los abordajes respectivos, y que las posibilidades realizables dentro de estos espacios han sido identificadas. En general, los fenómenos, las condiciones, los espacios y las posibilidades investigadas varían significativamente de uno a otro abordaje.

Los adeptos de un abordaje determinada quizá se sientan tentados a generalizar sus asertos: "Todo comportamiento se explica según los términos de las categorías conductistas", o "Todos los fenómenos mentales son computables". Estas propuestas son mutuamente contradictorias, pero ninguna de ellas puede aceptarse en concordancia con la imparcialidad. Ambas son propuestas metafísi- 
cas - generalizaciones muy distantes de tomar contacto con la evidencia empírica, motivadas (lo sostengo), en parte, por la adopción de valores específicos (Lacey, 2001b).

Existe conflicto entre abordajes tales como aquellos del conductismo y de la psicología cognitiva: conflicto para obtener los recursos necesarios para realizar investigaciones, competencia por puestos dominantes en la profesión, contradicción entre las perspectivas metafísicas asociadas - pero no hay contradicción entre los resultados empíricos y teóricos establecidos correctamente. Con todo, existe discordia sobre su valor, importancia o significado: Los resultados conductistas, al ser aplicados, favorecen especialmente a aquellos que desean aumentar nuestra capacidad de controlar el comportamiento humano; y tal deseo es rechazado por quienes dan énfasis a valores tales como la racionalidad y la libertad (Lacey, $2001 b)$. Pero, una vez más: aceptar una teoría que concuerde con la imparcialidad no implica una neutralidad aplicada. Los críticos del conductismo cuestionan su valor o su significado, en parte, debido a los intereses que serían favorecidos por las aplicaciones de sus resultados.

Para entender bien este tipo de situación, es importante reconocer que cada abordaje quiere tener acceso a cierta clase de posibilidades, generalmente de interés especial para una perspectiva valorativa en particular, (aunque existan, evidentemente, posibilidades que cubren una gama muy amplia de perspectivas de valor). Entonces, salvo excepciones muy importantes, los resultados de cada abordaje van a carecer de neutralidad aplicada. Por lo tanto, si quisiéramos sustentar el ideal de la neutralidad aplicada, necesitaríamos robustecer, en toda la disciplina, las condiciones para una investigación sustentada por múltiples abordajes (Lacey, 1999a: cap. 10). [Quiero observar también, sin entrar en muchas elaboraciones, que sin las condiciones para las investigaciones con muchos abordajes, sería muy fácil confundir una generalización metafísica (generalizada de teorías bien establecidas en ciertos dominios de fenómenos) con algo que es un resultado concordante con la imparcialidad. Entonces, se vuelve plausible el que tal propuesta metafísica fuese considerada como una restricción cognitiva impuesta correctamente sobre toda empresa científica].

El argumento a favor de múltiples discursos dentro de la ciencia quizá sea más obvio en lo tocante a las ciencias humanas. Puede ser hacerse, empero, en otras áreas de la ciencia, por ejemplo, la ciencia agrícola. (Aquí anticipo el argumento de mi segunda contribución a este libro.) En esta disciplina, ¿qué tipos de posibilidad queremos investigar? ¿Posibilidades abiertas para los productos de simientes transgénicas o posibilidades de aumento de productividad en los agroecosistemas sustentables —u otras (Lacey, 1998: cap. 6; 1999d; 200 lc)? Las posibilidades de las simientes transgénicas y de sus productos pueden investigarse en un abordaje dentro de la investigación sujeta a restricciones de corte galileano, las cuales ordenan representar los fenómenos en cuanto ley,

¿Cómo deben influir los valores en la ciencia? 
estructura (molecular) y proceso (bioquímico) subyacentes; las posibilidades de los agroecosistemas, en un abordaje que no abstraiga a los organismos de sus nichos agroecosistémicos, e investigue las relaciones y las interacciones entre los organismos y su medioambiente, del cual los organismos son partes integrantes que forman "totalidades" más o menos autoajustadas (Altieri, 1998). Un abordaje agroecológico podría estar e n continuidad con el conocimiento tradicional de una comunidad y de una cultura. Ahí, la empresa científica sistemática y empírica reconocería la importancia del papel de los agricultores locales al lado de los científicos "especializados".

Una vez más: la imparcialidad no confiere prioridad a uno de los abordajes en detrimento del otro - aunque, debido a razones sociales y prácticas podría haber muchos y diversos obstáculos que dificulten que los dos se suscitasen al mismo tiempo. Son los valores que fortalecen la clave para escoger entre uno u otro abordaje. Anteriormente decía que, a mi modo de ver, los abordajes galileanos lienen relaciones que refuerzan mutuamente los modos específicamente modernos de valorar el control de los objetos naturales: Dichos modos tienen que ver con el alcance del control y su falta de subordinación relativa a los demás valores sociales y morales, y con la percepción profunda de que el control es el modo de interacción humana característico de cara a la naturaleza, de tal manera que se valora mucho la expansión de las tecnologías (informadas por el conocimiento confirmado bajo abordajes galileanos) a más y más esferas de la vida, así como el empleo de tecnología avanzada para resolver más y más problemas (Lacey, 1998: caps. 5 y 6; 1999a: cap. 6; 1999d).

El énfasis biotecnológico en la agricultura está ligado a aquellos modos de valorar el control, y actualmente estos tienden a interpretarse a la luz de los objetivos de carácter global político-económico neoliberal. Por otro lado, los abordajes agrotecnológicos tienden a ser sensibles a valores tales como la sustentabilidad ecológica, bienestar de todas las personas, fortalecimiento de la comunidad como agente y justicia social —entendida en los términos de toda la gama de los derechos humanos universales (Lacey, 1998; caps. 1, 6 y 8; 1999: cap. 8).

Vuclvo a insistir: La adopción de una u otra de estas perspectivas valorativas no podría establecer cuáles son las posibilidades realizables, o aun si existen posibilidades de los tipos deseados. Eso podría establecerse solamente en el decurso de la investigación empírica. También, cada abordajc, por sí solo, carece de neutralidad aplicada — csto es, no produce resultados que podrían ser aplicados genéricamente de modo igual para todo el mundo: no sólo para aquellos que adopten valores neoliberales, sino también para quienes adopten los valores del movimiento popular. Los resultados cientílicos bien confirmados, obtenidos bajo las restricciones galileanas, son cognitivamente neutros; el lenguaje "tćcnico" empleado no ticne ningún papel valorativo y, por lo tanto, ninguna implicación en el dominio de los valores. Pero ello no es suficiente para la neutralidad aplicada. 
El empleo riguroso de las restricciones galileanas no es la garantía de que cierlos valores (aquellos que están ligados al control de los objetos naturales) no estén fundamentalmente en juego al emprender la investigación científica. Por lo lanto, quien niegue valor de legitimación a los otros abordajes de la investigación sistemática y empírica (tal como la agroecología) aduciendo que les lalta ajustarse a las restricciones galileanas, estaría, efeclivamente, incurriendo en la misma lógica que la iglesia utilizó contra Galileo.

III

¿Cómo deben influir los valores en la ciencia? Es claro que, a mi modo de ver, los valores y la ciencia deben interpenetrarse, que el abordaje adoptado en la investigación se verá afectado (en un grado significativo) por los valores (Barbosa de Oliveira, 1999: cap. 13). Yo quería sacar de mi discusión las siguientes conclusiones (aunque reconozco que el argumento que he ofrecido aquí dista de ser completo):

I. El compromiso con la imparcialidad es esencial: Acéptense teorías de un dominio específico si, y solamente si, manifiestan los valores cognitivos en un grado alto, siempre más alto que las teorías rivales (lo que implica que se les tiene que haber dado a esas teorías la oportunidad de desarrollarse). En la práctica, esto signifíca que es importante prestar una atención clara a los límites del poder que tiene una teoría para robustecer explicaciones e identificar las posibilidades de las cosas - y un escepticismo de cara a las tentativas de legitimar aplicaciones usando presupuestos discordantes de la imparcialidad (Lacey, 1999c). La imparcialidad representa el residuo fundamental de la propuesta de que los valores y la ciencia no deben interpenetrarse.

2. Manténgase el ideal de neutralidad aplicada, pese a las dificultades y tensiones generadas en la práctica cientílica (Lacey, 1999a: cap. 10). Permítase, en las áreas apropiadas, que se desarrollen múltiples abordajes, con la conciencia clara del modo por el cual un abordaje se liga inicialmente a los valores particulares —de tal manera que, en primer lugar, los valores no desempeñen un papel camuflado en la aceptación y en la recepción de teorías; en segundo lugar, las controversias sobre los valores se vuelven parte del discurso de la comunidad de investigadores en el mundo entero y los científicos tendrán la libertad de optar por aquellos abordajes que les permitan explorar, y talvez identificar, posibilidades que sirvan a intereses tales como, por ejemplo, aquellos que benefician a países pobres o a minorías (Lacey, 1998: cap. 1); y, en tercer lugar, la ciencia no se excluirá del dominio de la discusión democrática. [Cómo equilibrar la autonomía y la discusión democrática es un punto que necesita un mayor debate.]

3. Fortalézcase el compromiso con el "ethos" científico, con la tolerancia y la apertura a los abordajes vinculados a diferentes valores, para evitar que la

¿Cómo deben influir los valores en la ciencia? 
"especialización" no funcione como cobertura para una perspectiva de valores particular compartida (talvez, inconcientemente) por los investigadores (Lacey, 1999a: cap. 9). Está claro que la ciencia no debe tener lugar ni para las "auras" —pero tampoco para las "águilas"— que vuelan encima de toda crítica.

Hay que recordar que el águila es también el símbolo del poder imperial, el cual tolera poca oposición seria. En la ciencia, gracias a los "abordajes múltiples", abiertos a la gran variedad de posibilidades del mundo y de la vida humana, el águila debería coexistir con la golondrina que migra a través de los continentes, con el colibrí parado delante de una flor, con el papagayo fascinado con sus colores maravillosos, con el mochuelo que resiste la extinción aunque se destruya su medio ambiente, con la kookaburra riendo al nacer y al ver el sol, y con el canario encantando a todos con su trino.

En cuanto se insista en la imparcialidad de los juicios científicos, la interpenetración de los valores y la ciencia —el papel de los valores con respecto a la elección del abordaje que se seguirá- no impedirá que, en la investigación científica, se continúe obteniendo conocimientos bien fundamentados sobre las causas y las posibilidades de los fenómenos del mundo.

\section{REFERENCIAS BIBLIOGRÁFICAS}

Altieri. M. (1998): Agroecologia: A dinâmica produtiva da agricultura sustentável. Porto Alegre: Editora da Universidade Federal do Rio Grande do Sul.

Barbosa de Oliveira, M. (1999): Da Ciência Cognitiva a Dialética. Sāo Paulo: Discurso Editorial.

Blackwell, R. J.: (1991) Galileo and the Bible. Notre Dame: University of Notre Dame Press.

Cupani, A. (1998): "A propósito do «ethos» da ciência", Episteme 3: 16-38.

Finocchiaro, M. A.: Notes and Appendix to Galileo on the World Systems, a new abridged translation and guide. Berkeley: University of California Press.

Galilei, Galileo: (1613/1989): Letter to Castelli, in Finocciaro, M.A. (ed.): The Galileo A.ffair: A documentary history. Berkelye: University of California Press, 1989.

- (1615/1957): Letter to the Grand Duchess Christina, in S. Drake (ed.): Discoveries and Opinions of Galileo. Garden City (New Jersey): Doubleday.

- (1623/1957): The Assayer, in S. Drake (ed.): Discoveries and Opinions of Galileo. Garden City (New Jersey): Doubleday.

- _ (1632/1962) Dialogue Concerning the Two Chief World Systems, translated by S. Drake. Berkeley: University of California Press.

Lacey, H. (1998): Valores e atividade científica. Sāo Paulo: Editorial Discurso.

- (1999a): Is Science Value Free? Values and scientific understanding. London: Routledge.

(1999b): On cognitive and social values: A reply to my critics, Science and

Education 8: 89-103.

- (1999c): Values and the conduct of science: Principles, Principia 3: 57-85. (1999d): Science and Values (2): Manuscrito 22: 165-203. 
(2001a): The ways in which the sciences are and are not value free, in $\mathrm{P}$ Gardenfors, K. Kijania-Placek \& J. Wolenski (eds), Proceedings of the I $l^{\text {th }}$ International Congress of Logic, Methodology cind Philosophy of Science. Dordrecht: Kluwer (no prelo).

(2001 b): Psicologia Experimental e Natureza Humana. Florianópolis, S.C.: EdiIora da Universidade Federal de Santa Caterina (en preparación).

(2001 c): Incommesurability and 'multicultural science', in P. Hoyningen-Huene \& H. Sankey (eds), Incommesurability and Related Matters. Dordrecht: Kluwer- Boston Studies in the Philosophy of Science (no prelo).

Lacey, H. \& Mariconda, P. (2000), The eagle and the starlings: Galileo on the authonomy of science. Conferencia presentada en HOPOS 2000: Tercera Conferencia Internacional de Filosofía de la Ciencia, Círculo del Instituto de Viena, Universidad de Viena, Viena (Austria), 6 de julio de 2000.

Mariconda, P. (1997), A questão da autonomia da ciência em Galileo. Conferencia presentada al II Encuentro de Estudios Filosóficos del Siglo XVII, São Paulo, 26 de mayo de 1997.

Poincaré, H. (1920/1958) The Value of Science. New York: Dover.

¿Cómo deben influir los valores en la ciencia? 